\title{
BEXHI: A Mechanical Structure for Prototyping Bendable and EXpandable Handheld Interfaces
}

\author{
Michael Ortega \\ Université Grenoble-Alpes, LIG, CNRS \\ michael.ortega@imag.fr
}

\author{
Alix Goguey \\ Université Grenoble-Alpes, LIG \\ alix.goguey@univ-grenoble-alpes.fr
}

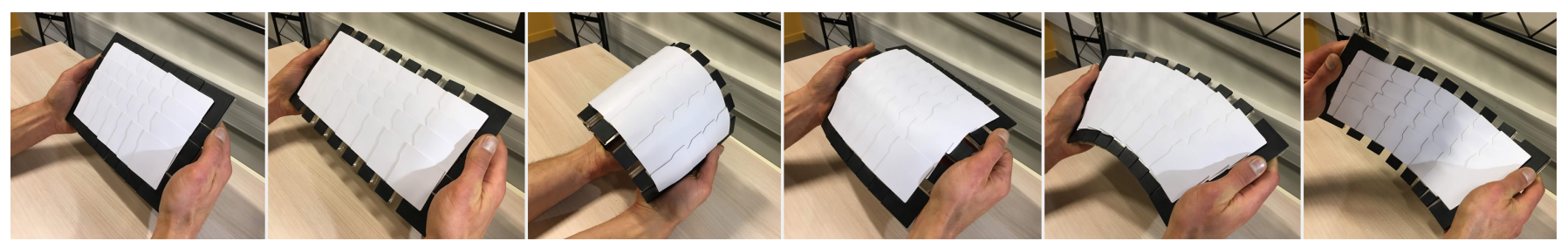

Figure 1: Six example shapes of a BEXHI prototype: a tablet that can be expanded and bent in 2 dimensions.

\begin{abstract}
In this paper, we present BEXHI, a new mechanical structure for prototyping expandable and bendable handheld devices. Many research projects have pushed bendable surfaces from prototypes to commercially viable devices. In the meantime, expandable devices have become a topic of interest letting one foresee that such devices are on the horizon. With BEXHI, we provide a structure to explore the combined capabilities of these devices. The structure consists of multiple interweaved units allowing non-porous expandable surfaces to bend. Through an instanciation, we illustrate and discuss that the BEXHI structure allows for the exploration of the combination of both bend and expansion interaction spaces.
\end{abstract}

\section{Author Keywords}

Shape Changing Interface; Organic User Interface; Bending gesture; Expansion.

\section{INTRODUCTION}

From mobile phones to tablets, we now use interactive surfaces on a daily basis empowering us to complete many of our tasks. The current evolution of our lifestyle will, arguably, have the tendency to reinforce our nomadic usage of devices. To improve the interaction bandwidth of our devices, researchers are seeking to break the expressiveness ${ }^{1}$ barrier. One way of doing so is to make our devices more 'organic', through Shape

\footnotetext{
${ }^{1}$ Expressiveness refers to the number of modalities used to transmit the input vocabulary. The greater the number, the more expressive the device.
}

Permission to make digital or hard copies of all or part of this work for personal or classroom use is granted without fee provided that copies are not made or distributed for profit or commercial advantage and that copies bear this notice and the full citation on the first page. Copyrights for components of this work owned by others than ACM must be honored. Abstracting with credit is permitted. To copy otherwise, or republish, to post on servers or to redistribute to lists, requires prior specific permission and/or a fee. Request permissions from permissions@acm.org.

ISS '19, November 10-13, 2019, Daejeon, Republic of Korea

(C) 2019 ACM. ISBN 978-1-4503-6891-9/19/11 . \$15.00

DOI: https://doi .org/10.1145/3343055.3359703
Changing Interfaces (SCI) [2]. Organic User Interfaces (OUI) [23] are SCIs in which the display is an input, that changes its shape through manual/automated deformations, enriching the interaction vocabulary.

There are many directions explored from screen(s) reconfiguration, such as Cubimorph [17] or Doppio [19], to bendable screens, such as Codex [8] or FoldMe [9]. The latter category even starts to be commercially viable, for instance with the Huawei Mate X and the Samsung Galaxy Fold. The technology is catching up with research concepts, and fully bendable screens are on the horizon. Concurrently, new features are studied, such as expansion. Expandable surfaces, such as Xpaaand [10] or EXHI-Bit [12], aim at dynamically changing their form factor by modifying the area of the screen realestate. It is important to note that both the expansion and bending features are orthogonal (ie. they can be combined), and increase surfaces expressiveness. These surfaces are already studied through user elicitation studies based on videos, highlighting a need for physical tools [13].

In this paper we use these observations as a premise, and aim to provide the research community with a mechanical structure for exploring devices that combine expansion and bending. We propose BEXHI: an upgraded version of EXHI-Bit. We provide a thorough description of the BEXHI structure, as well as an instantiation of it (a bendable and expandable tablet). We also provide all the digital material needed to build and easily replicate such a device. Finally, we discuss the possibilities enabled by BEXHI through possible applications.

\section{RELATED WORK}

Deformable surfaces is a well known topic in the shape changing interfaces literature. It has been tackled through different angles, whether through the deformation of a surface casing (eg. [14]) or of its screen. This section is an overview of works focusing on deformable screens through bending and expansion only. Due to their nature, ie. bending along predefined axis, we do not consider folding. 


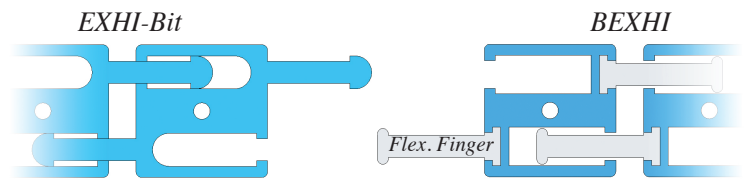

Figure 2: Plexiglas Interweaving layers: EXHI-Bit (left) vs BEXHI $($ right $)$. BEXHI fingers are printed, in TPU 95A, making them flexible.

Bendable screens, of which Gummi [18] is one of the firsts, can be deformed along an infinite amount of axes. In some cases, like HoloFlex [6], all the axes are parallel, along one edge. This type of device was studied by Ahmaniemi et al. who investigated which kind of control should these bending gestures be used for [1]. In other researches, multiple bends can be performed simultaneously along any axis of the device [20]. Even though the screen itself is not bendable, FlexCase provides several examples of how these bending gestures could be used as inputs (eg. map navigation, gaming, ...) [15]. Similar to [1], Girouard et al. investigated one-handed bend gestures and emit recommendations on which gestures to prefer and use [3].

About expansion, a thorough survey can be found in [12], which also presents EXHI-Bit, the recent work we built on.

Due to the current lack of prototyping structures, combination of both bending and expansion has not been explicitly explored yet. As precursors, we can consider the 3 following works. PaperFold is a prototype device using detachable screens that cannot only be connected or broken apart to modify the screen footprint, but also dynamically folded along their bezels [5]. DisplayStacks are fully bendable screens whose screens can be stacked or slid over [4]. They are used to contextualize views on documents, as well as expand the view port in one dimension. Similarly, PaperTab [22] is a tablet composed of multiple bendable touchscreens. However, a mechanical structure for allowing both bending and expansion in a integrated way was still missing.

\section{BEXHI}

For creating BEXHI, ie. integrating bending into EXHI-Bit, both the interweaving mechanism and the covering system were redesigned. These aspects are presented in the following, after a short description of EXHI-Bit. Figure 5 resumes the difference between an EXHI-Bit unit and a BEXHI one.

\section{Starting point: EXHI-Bit}

EXHI-Bit is an aggregation of identical elementary units. Each unit is mechanically connected up to 4 other units, and can slide away from the others. A unit is a sandwich of plexiglas layers, topped by a paper tile. Two of these layers, called interweaving layers (Figure 2left), ensure connection with the neighbours (see Figure 5 in [12]). The paper tile ensures the non-porosity [16] of the complete surface. An external projector and a tracking system are used to display digital content.

Considering bending interaction, an EXHI-Bit surface has not enough flexibility. Plexiglas is too brittle, and the paper tiles do not stick the surface when bent.

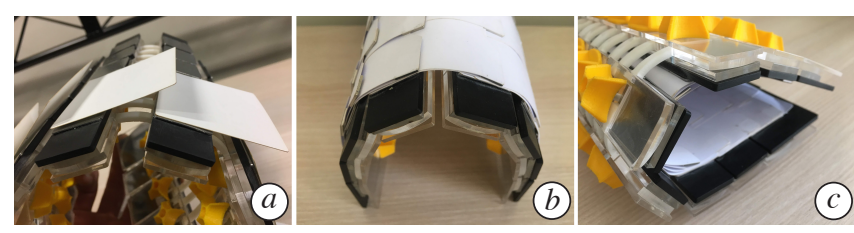

Figure 3: $a$ : EXHI-Bit tiles (simple paper squares) lift away from the surface when it is bent. $b, c$ : BEXHI tiles (with magnets) allow bending in both directions without lifting.

\section{BEXHI Interweaving Mechanism}

Starting from the layering principle of EXHI-Bit units, we propose to keep the main core of a unit rigid, and to support bending through the fingers of the interweaving layers only.

We first tested to laser cut bending-cut patterns on each finger, but after testing and due to the short nature of the fingers, plexiglas was still too brittle to support marked bending movements. Moreover, when bent, such fingers do not slide smoothly into the neighbor units. We then looked for another material, and in order to keep the sliding properties between the units, we opted to change the material for the fingers only. We kept using plexiglas for the central part. In BEXHI, the fingers are 3D printed with flexible plastic (TPU 95A), and locked in force into the central part using notches that maintain them in place (Figure 2). Two neighbouring units can be easily bent beyond 90 degrees, and the TPU 95A plastic is strong enough to endure bending stress ${ }^{2}$.

\section{BEXHI Covering Mechanism}

EXHI-Bit paper tiles are not adapted to bendable surfaces, especially when bending up, $i e$. screen center moving towards the user. First, as shown in Figure $3 a$, the un-pasted parts of the tiles lift away from the surface, breaking the surface continuity. Next, due to the extra length implied by a curved junction between two tiles, the size of the interstice can be larger than the size of one unit. To keep the non-porosity property, the tiles should then be larger than two units, creating interferences with their neighbouring tiles.

Two questions have then to be answered : (1) How to make the tiles stick to the surface while it is bent, and (2) how to make larger tiles while avoiding collisions ?

\section{Keeping tiles pasted on the surface}

We first tried fabric. However, as in [12], we were left with rigidity issues: it wrinkled when bending down (screen center going away from the user) and tessellated when bending up. We then came back to paper, which already have good intrinsic bending properties, and opted to improve the fixing principles by using a magnets. Tiles in silicon or flexible plastic could work as well, however depending on the tracking system used, reflexion properties of these material might cause interferences.

We first added a thin metallic layer atop each unit, under the paper tile (Figure 5). Next, we placed magnets under the corner opposite to the one fixing the tiles to their unit (Figures 5 and 4). The metallic layer allows tiles, augmented with

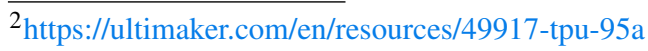



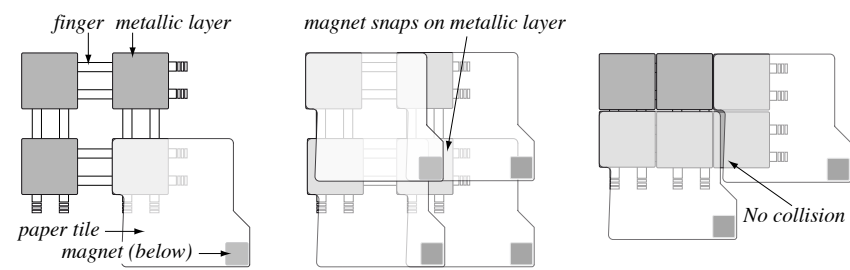

Figure 4: Top view of BEXHI tiles. Left: Top-left part of a tile is pasted on the metallic layer of a unit. middle: the magnet of a tile is always up to a metallic layer. right: When the surface is closed, the new design avoid collision between two separated tiles.

magnets, to be maintained on the neighbouring units while still being free to slide (Figure 4middle). The magnets strength is a careful trade-off between avoiding tiles lifting when the surface bends and allowing the manipulation of both expansion and bending with a reasonable user's force. Border units have one more magnet along the bezel to help maintaining the tiles on the surface edge, and only one tile has three magnets: the corner tile that covers three neighbour units. As shown in Figure 1, the tiles adopt the surface curvature and do not lift away even with an extreme bending.

\section{Avoiding tiles collision}

As mentioned above, due to the combination of bending and expansion, the interstice between two units can be larger than the size of one unit. Compared to EXHI-Bit specifications, BEXHI tiles have to be larger than twice the size of the unit to: (1) completely hide the interstice, and (2) always touch the neighbour unit's top and avoid lifting away. However, increasing the size of the tiles creates collisions between tiles of one unit and their second next neighbours (two units further). The design of the tile has to be adapted as it cannot keep its square shape. Figure 4 shows the new tiles design. This design avoids tiles collisions by enlarging the tiles at magnet position, $i e$. bottom right corner, and reducing the tiles at the opposite edge. Finally, by keeping the overlapping configuration of EXHI-Bit, these modifications do not have to be adapted for the vertical dimension.

\section{Manipulation}

When we manipulated a BEXHI structure for the first time, we observed that when manipulating the tablet instance (next section), and more particularly expansion, one had to fight against the sliding property of the bezels (made in plexiglas). Moreover, local expansion or bending, ie. on a sub-part of a BEXHI structure only, is quite difficult to perform without handles. We iterated and added a new layer at the bottom of each BEXHI unit, fixed by the main screw. As shown in Figures $3 c$ and 5, this is a printed element (yellow in the figures), whose shape allows the user to grip a single unit in any direction.

After an empirical evaluation, we observed that users were confident in handling and manipulating the tablet instance.

Finally, the structure is not particularly tied to a rectangle form factor. More exotic form factors like the ones presented in [12] could be supported. However, bending can introduce some suppleness.

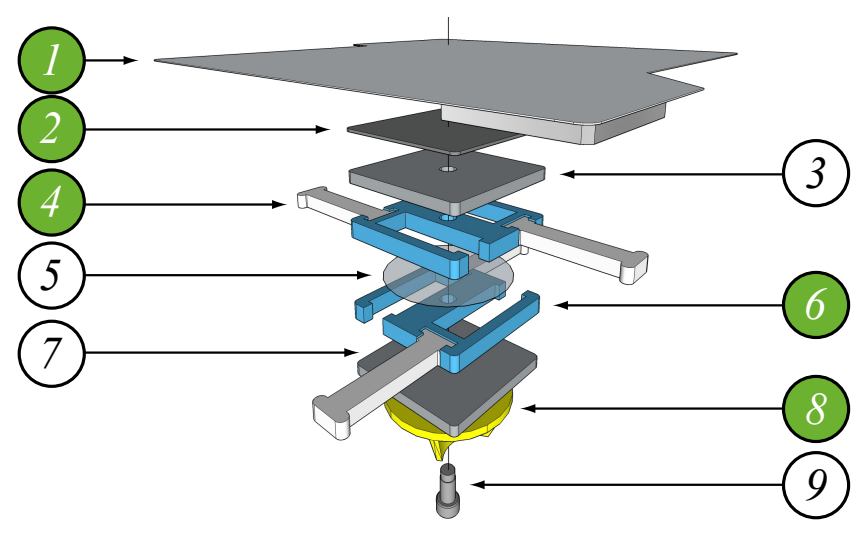

Figure 5: Layers for a BEXHI unit. Green highlights contributions. 1: Paper tile with a magnet at the corner. 2: Metallic layer on which magnets keep stick. 3,7: Plexiglas layer that sandwiches the interweaving parts. 4,6: Interweaving parts. 5: Plastic disk that improves sliding and avoids interlocking. 8: Printed grip for manipulation. 9: Screw, that only screws in layer 3, making a rotation axis for layers 4 to 8 .

\section{INSTANTIATION}

Using these design improvements and to illustrate the BEXHI concept, we created an expandable and bendable tablet prototype (Figure 1). In this section, we first describe the material used. Next, to better grasp the physical capabilities of the tablet, we present its characteristics using the Morphees vocabulary [16]. Finally, we present the display option we chose to implement.

In the following, the $\mathbf{x}$ axis is along the longest tablet edge (in landscape mode), then $\mathbf{y}$ axis is along the shortest edge.

\section{Configuration}

Considering $n_{x}$ and $n_{y}$ as the number of units along $x$ and $y$ respectively, $n_{x}=8 \quad n_{y}=5$ in this prototype. A unit edges are $L=2.4 \mathrm{~cm}$ large each. Bezel units are larger: $5 \mathrm{~cm}$. Their 'screen' surface is also $2.4 \times 2.4 \mathrm{~cm}$ large, and the rest is used for holding the tablet (Figure 1). The expansion between two units, $g$ (for gap), varies from 0 to $1.6 \mathrm{~cm}$.

This prototype uses neodymium squared magnets, $1 \mathrm{~mm}$ thick, with a strength of $0.5 \mathrm{~kg} / \mathrm{cm}^{2}$. Grips are printed with PLA, plexiglas is $3 \mathrm{~mm}$ thick, and the paper grammage is $250 \mathrm{~g} / \mathrm{m}^{2}$. All the digital material needed to replicate this tablet can be found at alixgoguey.fr/BEXHI.

\section{Curvature}

As in Morphees [16], the curvature is computed using 3 consecutive control points: in our case, units are 2D surfaces. A flat surface corresponds to a 0 curvature, \pm signs refer to the bending direction. When bending up (ie. surface centre moves towards the user), magnets start to pop from the neighbouring metallic layers at $0.6 \pi$ (the angle between the planes defined by the 2 external control points / units), making the effect in figure $3 a$. When bending down, the curvature can reach $-\pi$ (the 2 planes are parallel), as shown in Figure $3 c$.

\section{Area}

The screen area (without the bezel) is computed as follow (with $g$ representing the gap size between two units):

$$
A\left(g_{x}, g_{y}\right)=\left[n_{x} \cdot L+\left(n_{x}-1\right) \cdot g_{x}\right] \cdot\left[n_{y} \cdot L+\left(n_{y}-1\right) \cdot g_{y}\right]
$$


with $A_{\min }=A(0,0)=252 \mathrm{~cm}^{2}$ and $A_{\max }=A(1.6,1.6)=$ $594 \mathrm{~cm}^{2}$ in our prototype.

\section{Zero-crossing}

While bending along the x-axis, a maximum of 3 zerocrossings can be performed. However, in practice and in order to allow room for manipulation, up to 2 zero-crossings are feasible. Along the y-axis there are a maximum of 2. Again, such a shape is difficult to reach, especially with fingers on the tablet left and right sides. Only 1 zero-crossing should then be considered for interaction along the y-axis.

\section{Stretchability}

Strechability is computed as follow:

$$
S\left(g_{x}, g_{y}\right)=100 \cdot\left[A\left(g_{x}, g_{y}\right)-A_{\min }\right] / A_{\min }
$$

with $S_{x}=S(1.6,0)=56 \%$ (being the maximum expansion along the x axis), $S_{y}=S(0,1.6)=50 \%$ (y axis) and $S_{\max }=$ $S(1.6,1.6)=135 \%$ (both axes)in our prototype.

\section{Display}

As in EXHI-Bit [12], we display digital content by projecting on the tablet. We track the contour of the surface with an Optitrack system, and project our digital content using an external projector. There are other possible solutions arguably less expensive, such as Flexpad (cheaper depth sensor to track a flexible surface) [20], or more accurate, such as Narita et al. work [11]. The EXHI-Bit solution is a good compromise between precision and algorithmic complexity, allowing fast prototyping of new applications.

\section{POSSIBLE APPLICATIONS}

In the following, we provide few examples, based on our BEXHI instantiation, of how both bending and expansion can be used in complementary and/or concurrent ways.

\section{Manipulating a slider}

In many works, such as ReFlex [21] or TouchMark [24], bending a side of a screen up or down allows to change a linear value. However, the control is either relative and discrete (eg. bending up/down to go to the next/previous page) or has a fixed precision due to the bending capabilities of the screen. Using expansion allows for an adaptable granularity of the bending precision: the expansion can increase or decrease the surface area which in turn changes the minimum amount of bending that can be performed. For instance, one can implement a slider whose value is changed with a unit precision when there is no expansion, allowing for a fast and rough value setting, like the ballistic phase of a pointing movement, and change the value with a 0.1 precision when the tablet is expanded (see the accompanying video).

\section{Pan and zoom on a map}

In the previous example, expansion is used as a modifier for bending (eg. giving bending gestures slightly different meaning). However, one could imagine using both type of gesture without some kind of hierarchy, and allowing both to be used concurrently. For instance, one can use a the following gestures for panning a map: expanding the top (resp. bottom) bezel only to pan upwards (resp. downwards) and bending the right (resp. left) bezel to pan rightwards (resp. leftwards).
These gestures (and their combinations) can all be performed with both hands always gripping the side bezels. Achieving the same functionalities with expansion only or bend only is arguably more difficult without changing the grip, even more so if you add a zoom control. In our example zoom in/out could be achieve by bending down/up the surface. This simple example illustrates the increase in expressiveness even when keeping hands movement minimal.

\section{Watching a movie}

The complementarity of bending and expansion features can also be illustrated in a 'watching a movie' scenario. While watching a movie on a BEXHI tablet, the viewer can expand the screen to watch more comfortably, but also bend it slightly and place it vertically on a table enabling the screen to self stands (see the accompanying video).

Those simple examples aim at highlighting the interaction possibilities, but elude that many design choices are needed. For instance, while manipulating the slider, should the movement and change of value be mapped absolutely or relatively? Should the bend up increase or decrease the value? Should the bending movements be symmetric (bending both screen sides at the same time)? We argue that many exciting research about interaction techniques could come out from BEXHI.

\section{CONCLUSION, LIMITATIONS AND FUTURE WORKS}

We presented BEXHI, a new mechanical structure solution for prototyping bendable and expandable devices. While viable technologies are catching up with research concepts of deformable surfaces and screens, we aimed at pushing further the barrier by providing the research community with a new tool to explore and design new exciting interactive systems. As illustrated by the tablet instantiation and the three possible applications, BEXHI enables the exploration of new interactions combining two dimensions that have been explored separately. Our hope with BEXHI, is that this work will further stimulate OUI research and fill the need identified in [13].

Next steps include investigating actuation. As for other works on bendable or expandable screens, adding a bending capacity imposes on the screen an inevitable bent under its own weight if not gripped properly. This side-effect also renders the expansion gesture hard to achieve if, again, the grip is not carefully placed. We argue that these limitation could be alleviated with actuation, using material solutions like HotFlex [7], where 3D printer material is augmented with heat actuators to dynamically adapt the stiffness of printed objects. Our printed fingers could be improved to ease the tablet manipulation.

Investigations will also be made on the display aspect. While prototyping interactions using projection is an efficient, easy and fast to implement solution, it is not adapted to real-world mobility contexts of use (eg. interaction while walking). We'll investigate the replacement of the paper tiles with OLED screens.

\section{ACKNOWLEDGMENTS}

This work is supported by the French National Research Agency: ANATOMY2020 project (ANR-16-CE38-0011). 


\section{REFERENCES}

[1] Teemu T. Ahmaniemi, Johan Kildal, and Merja Haveri. 2014. What is a Device Bend Gesture Really Good for?. In Proceedings of the 32Nd Annual ACM Conference on Human Factors in Computing Systems (CHI'14). ACM, New York, NY, USA, 3503-3512. DOI : http://dx.doi.org/10.1145/2556288.2557306

[2] Marcelo Coelho and Jamie Zigelbaum. 2011. Shape-changing Interfaces. Personal Ubiquitous Comput. 15, 2 (Feb. 2011), 161-173. DOI : http://dx . doi . org/10.1007/s00779-010-0311-y

[3] Audrey Girouard, Jessica Lo, Md Riyadh, Farshad Daliri, Alexander Keith Eady, and Jerome Pasquero. 2015. One-Handed Bend Interactions with Deformable Smartphones. In Proceedings of the 33rd Annual ACM Conference on Human Factors in Computing Systems (CHI '15). ACM, New York, NY, USA, 1509-1518. DOI : http://dx.doi.org/10.1145/2702123.2702513

[4] Audrey Girouard, Aneesh Tarun, and Roel Vertegaal. 2012. DisplayStacks: Interaction Techniques for Stacks of Flexible Thin-film Displays. In Proceedings of the SIGCHI Conference on Human Factors in Computing Systems (CHI'12). ACM, New York,NY,USA, 2431-2440. DOI: http://dx.doi.org/10.1145/2207676.2208406

[5] Antonio Gomes and Roel Vertegaal. 2015. PaperFold: Evaluating Shape Changes for Viewport Transformations in Foldable Thin-Film Display Devices. In Proceedings of the Ninth International Conference on Tangible, Embedded, and Embodied Interaction (TEI'15). ACM, New York, NY, USA, 153-160. DOI : http://dx.doi.org/10.1145/2677199.2680572

[6] Daniel Gotsch, Xujing Zhang, Juan Pablo Carrascal, and Roel Vertegaal. 2016. HoloFlex: A Flexible Light-Field Smartphone with a Microlens Array and a P-OLED Touchscreen. In Proceedings of the 29th Annual Symposium on User Interface Software and Technology (UIST '16). ACM, New York, NY, USA, 69-79. DOI : http://dx.doi.org/10.1145/2984511.2984524

[7] Daniel Groeger, Elena Chong Loo, and Jürgen Steimle. 2016. HotFlex: Post-print Customization of 3D Prints Using Embedded State Change. In Proceedings of the $2016 \mathrm{CHI}$ Conference on Human Factors in Computing Systems (CHI '16). ACM, New York, NY, USA, 420-432. DOI:http://dx . doi .org/10.1145/2858036.2858191

[8] Ken Hinckley, Morgan Dixon, Raman Sarin, Francois Guimbretiere, and Ravin Balakrishnan. 2009. Codex: A Dual Screen Tablet Computer. In Proceedings of the SIGCHI Conference on Human Factors in Computing Systems (CHI '09). ACM, New York, NY, USA, 1933-1942. DOI : http://dx . doi .org/10.1145/1518701.1518996

[9] Mohammadreza Khalilbeigi, Roman Lissermann, Wolfgang Kleine, and Jürgen Steimle. 2012. FoldMe: Interacting with Double-sided Foldable Displays. In Proceedings of the Sixth International Conference on Tangible, Embedded and Embodied Interaction (TEI '12). ACM, New York, NY, USA, 33-40. DOI : http://dx.doi.org/10.1145/2148131.2148142

[10] Mohammadreza Khalilbeigi, Roman Lissermann, Max Mühlhäuser, and Jürgen Steimle. 2011. Xpaaand: Interaction Techniques for Rollable Displays. In Proceedings of the SIGCHI Conference on Human Factors in Computing Systems (CHI '11). ACM, New York, NY, USA, 2729-2732. DOI :

http://dx.doi.org/10.1145/1978942.1979344

[11] G. Narita, Y. Watanabe, and M. Ishikawa. 2017. Dynamic Projection Mapping onto Deforming Non-Rigid Surface Using Deformable Dot Cluster Marker. IEEE Transactions on Visualization and Computer Graphics 23, 3 (March 2017), 1235-1248. DOI : http://dx.doi.org/10.1109/TVCG.2016.2592910

[12] Michaël Ortega, Jérôme Maisonnasse, and Laurence Nigay. 2017. EXHI-bit: A Mechanical Structure for Prototyping EXpandable Handheld Interfaces. In Proceedings of the 19th International Conference on Human-Computer Interaction with Mobile Devices and Services (MobileHCI '17). ACM, New York, NY, USA, Article 4, 11 pages. DOI : http://dx. doi .org/10.1145/3098279.3098533
[13] Esben W. Pedersen, Sriram Subramanian, and Kasper Hornbæk. 2014 Is My Phone Alive?: A Large-scale Study of Shape Change in Handheld Devices Using Videos. In Proceedings of the SIGCHI Conference on Human Factors in Computing Systems (CHI '14). ACM, New York, NY, USA, 2579-2588. DOI: http://dx.doi.org/10.1145/2556288.2557018

[14] Majken Kirkegård Rasmussen, Timothy Merritt, Miguel Bruns Alonso, and Marianne Graves Petersen. 2016. Balancing User and System Control in Shape-Changing Interfaces: A Designerly Exploration. In Proceedings of the TEI '16: Tenth International Conference on Tangible, Embedded, and Embodied Interaction (TEI '16). ACM, New York, NY, USA, 202-210. DOI : http://dx .doi.org/10.1145/2839462.2839499

[15] Christian Rendl, David Kim, Patrick Parzer, Sean Fanello, Martin Zirkl, Gregor Scheipl, Michael Haller, and Shahram Izadi. 2016. FlexCase: Enhancing Mobile Interaction with a Flexible Sensing and Display Cover. In Proceedings of the 2016 CHI Conference on Human Factors in Computing Systems (CHI '16). ACM, New York, NY, USA, 5138-5150. DOI : http://dx.doi .org/10.1145/2858036.2858314

[16] Anne Roudaut, Abhijit Karnik, Markus Löchtefeld, and Sriram Subramanian. 2013. Morphees: Toward High "Shape Resolution" in Self-actuated Flexible Mobile Devices. In Proceedings of the SIGCHI Conference on Human Factors in Computing Systems (CHI '13). ACM, New York, NY, USA, 593-602. DOI : http://dx.doi .org/10.1145/2470654.2470738

[17] A. Roudaut, D. Krusteva, M. McCoy, A. Karnik, K. Ramani, and S. Subramanian. 2016. Cubimorph: Designing modular interactive devices. In 2016 IEEE International Conference on Robotics and Automation (ICRA). 3339-3345. DOI : http://dx.doi.org/10.1109/ICRA. 2016.7487508

[18] Carsten Schwesig, Ivan Poupyrev, and Eijiro Mori. 2004. Gummi: A Bendable Computer. In Proceedings of the SIGCHI Conference on Human Factors in Computing Systems (CHI '04). ACM, New York, NY, USA, 263-270. DOI:

http://dx.doi.org/10.1145/985692.985726

[19] Teddy Seyed, Xing-Dong Yang, and Daniel Vogel. 2016. Doppio: A Reconfigurable Dual-Face Smartwatch for Tangible Interaction. In Proceedings of the 2016 CHI Conference on Human Factors in Computing Systems (CHI '16). ACM, New York, NY, USA, 4675-4686. DOI :http://dx.doi.org/10.1145/2858036.2858256

[20] Jürgen Steimle, Andreas Jordt, and Pattie Maes. 2013. Flexpad: Highly Flexible Bending Interactions for Projected Handheld Displays. In Proceedings of the SIGCHI Conference on Human Factors in Computing Systems (CHI'13). ACM, New York, NY, USA, 237-246. DOI : http://dx . doi . org/10.1145/2470654 . 2470688

[21] Paul Strohmeier, Jesse Burstyn, Juan Pablo Carrascal, Vincent Levesque, and Roel Vertegaal. 2016. ReFlex: A Flexible Smartphone with Active Haptic Feedback for Bend Input. In Proceedings of the TEI '16: Tenth International Conference on Tangible, Embedded, and Embodied Interaction (TEI '16). ACM, New York, NY, USA, 185-192. DOI : http://dx.doi .org/10.1145/2839462 . 2839494

[22] Aneesh P. Tarun, Peng Wang, Audrey Girouard, Paul Strohmeier, Derek Reilly, and Roel Vertegaal. 2013. PaperTab: An Electronic Paper Computer with Multiple Large Flexible Electrophoretic Displays. In CHI' 13 Extended Abstracts on Human Factors in Computing Systems (CHI EA '13). ACM, New York, NY, USA, 3131-3134. DOI : http://dx.doi.org/10.1145/2468356.2479628

[23] Roel Vertegaal and Ivan Poupyrev. 2008. Introduction. Commun. ACM 51, 6 (June 2008), 26-30. DOI : http://dx.doi.org/10.1145/1349026.1349033

[24] Doug Wightman, Tim Ginn, and Roel Vertegaal. 2010. TouchMark: Flexible Document Navigation and Bookmarking Techniques for e-Book Readers. In Proceedings of Graphics Interface 2010 (GI'10). Canadian Information Processing Society, Toronto, Ont., Canada, Canada, 241-244. http://dl.acm.org/citation. cfm?id=1839214.1839256 